\title{
Type 2 Diabetes and Breast Cancer: The Interplay between Impaired Glucose Metabolism and Oxidant Stress
}

\author{
Patrizia Ferroni, ${ }^{1}$ Silvia Riondino, ${ }^{1,2}$ Oreste Buonomo, ${ }^{3}$ \\ Raffaele Palmirotta, ${ }^{1}$ Fiorella Guadagni, ${ }^{1}$ and Mario Roselli ${ }^{2}$ \\ ${ }^{1}$ San Raffaele Rome University, IRCCS San Raffaele Pisana, Research Center, Via di Val Cannuta 247, 00166 Rome, Italy \\ ${ }^{2}$ Department of Systems Medicine, Medical Oncology, Tor Vergata Clinical Center, University of Rome Tor Vergata, \\ Viale Oxford 81, 00133 Rome, Italy \\ ${ }^{3}$ Department of Surgery, Division of Surgical Oncology, Tor Vergata Clinical Center, University of Rome Tor Vergata, \\ Viale Oxford 81, 00133 Rome, Italy
}

Correspondence should be addressed to Mario Roselli; mario.roselli@uniroma2.it

Received 8 January 2015; Accepted 28 May 2015

Academic Editor: Amina El Jamali

Copyright (C) 2015 Patrizia Ferroni et al. This is an open access article distributed under the Creative Commons Attribution License, which permits unrestricted use, distribution, and reproduction in any medium, provided the original work is properly cited.

\begin{abstract}
Metabolic disorders, especially type 2 diabetes and its associated complications, represent a growing public health problem. Epidemiological findings indicate a close relationship between diabetes and many types of cancer (including breast cancer risk), which regards not only the dysmetabolic condition, but also its underlying risk factors and therapeutic interventions. This review discusses the advances in understanding of the mechanisms linking metabolic disorders and breast cancer. Among the proposed mechanisms to explain such an association, a major role is played by the dysregulated glucose metabolism, which concurs with a chronic proinflammatory condition and an associated oxidative stress to promote tumour initiation and progression. As regards the altered glucose metabolism, hyperinsulinaemia, both endogenous due to insulin-resistance and drug-induced, appears to promote tumour cell growth through the involvement of innate immune activation, platelet activation, increased reactive oxygen species, exposure to protumorigenic and proangiogenic cytokines, and increased substrate availability to neoplastic cells. In this context, understanding the relationship between metabolic disorders and cancer is becoming imperative, and an accurate analysis of these associations could be used to identify biomarkers able to predict disease risk and/or prognosis and to help in the choice of proper evidence-based diagnostic and therapeutic protocols.
\end{abstract}

\section{Introduction}

Type 2 diabetes (T2D) constitutes a growing public health problem, with a global prevalence of $8.3 \%$ in 2013 (undiagnosed in approximately $30 \%$ of the cases), which is estimated to rise to more than $10 \%$ by 2035 . Noteworthy, the largest increases will take place in developing countries, as T2D is epidemic in many low- and middle-income countries [1]. Although progression is not inevitable, impaired glucose tolerance (IGT, generally referred to as "prediabetes") is a key condition in the evolution toward T2D. In 2013, global IGT prevalence has been estimated at $6.9 \%$, a rate that is calculated to go up to $8 \%$ by 2035 .

The relevance of these figures is evident if one considers that T2D is associated with a decrease of health-related quality of life and overall life expectancy and that T2D remains one of the leading causes of death, worldwide (especially due to cardiovascular complications). Looking at the 2013 estimates, in fact, T2D accounted for $8.4 \%$ of global all-cause mortality among individuals aged between 20 and 79 years, with an $11 \%$ increase over previous estimates for 2011 [1]. To further worsen this picture is the epidemiologic evidence of a close relationship between T2D and increased cancer risk [2-4], although an accurate assessment of cancer risk is complicated by the occurrence of several confounding factors such as disease duration, varying metabolic profiles, and the possible presence of shared cancer-promoting factors [5]. Accordingly, the American Association of Clinical Endocrinologists and the American College of Endocrinology highlighted in a recent joint consensus report the need 
for systemic studies to further investigate this relationship (AACE/ACE Consensus Statement) [6].

\section{T2D and Breast Cancer Risk}

T2D may affect multiple organs in different ways, and the female breast is not an exception. Of particular interest is a peculiar condition known as "diabetic mastopathy," an infrequent proliferation of fibrous tissue in the breast parenchyma manifesting as unilateral or bilateral nodules [7]. Firstly described in 1984, diabetic mastopathy is a poorly characterized condition, often mistreated and not diagnosed as a diabetes complication. To date, there is no evidence of an association with breast cancer (BC), as the isolated case-reports of coexisting diabetic mastopathy and BC could merely represent two facets of concomitant diseases with high prevalence [7].

Beside this particular condition, increasing evidence suggest that diabetes contributes to BC risk. Up to $16 \%$ of BC patients, in fact, have T2D, which, in turn, has been associated with a $10-20 \%$ excessive risk of BC [8]. Moreover, several evidences indicate that $\mathrm{T} 2 \mathrm{D}$ and impaired glucose tolerance may worsen $\mathrm{BC}$ prognosis $[9,10]$. These figures were confirmed in a recent meta-analysis indicating an increased risk for BC of $23 \%$ in patients with T2D, and a $38 \%$ higher cancer-specific mortality risk in patients with T2D and BC. Accordingly, a positive association between $\mathrm{T} 2 \mathrm{D}$ and $\mathrm{BC}$ incidence and mortality during a 10-year follow-up was also reported [11].

The possibility that an impaired glucose metabolism may influence BC incidence and outcomes clearly has major implications for primary and secondary prevention of BC. In this respect, it has been recently suggested that the variable prevalence of dysmetabolic conditions across Europe may contribute, at least in part, to the variations in BC survival across the continent, which cannot be completely explained by differences in stage at diagnosis or patient management $[12,13]$.

Based on molecular expression, BC can be classified into different subtype, either expressing hormone receptors, ER (oestrogen receptor), PR (progesterone receptor), and growth factor receptor HER2 (Human Epidermal Growth Factor Receptor) [14]. Clinical studies demonstrated that triple-negative BCs are associated with the poorest prognosis. In this regard, an independent association between HER2 and both hyperglycaemia and insulin resistance has been found [15], and circulating HER-2 concentrations seem to be significantly increased in T2D patients [16].

All these factors may act through independent and/or synergic mechanisms, either being responsible for a metabolic, hormonal, and inflammatory interplay (responsible for the more general association with the metabolic syndrome, as in the case of colorectal cancer [17]), or by acting in a site-specific manner, as in the case of female reproductive organs, and specifically in breast.

\section{Impaired Glucose Regulation and Breast Cancer}

The possibility of a causal link between impaired glucose metabolism and cancer was initially raised by Marble in the first half of the 20th century [18]. Since then, several studies have investigated the possible etiological mechanisms underlying this association, demonstrating the central role of sustained hyperglycaemia, hyperinsulinemia, insulin resistance (IR), and hyperinsulinemia-related increase of insulin-like growth factor-1 (IGF-1) in cancer promotion and progression [19-22]. In turn, poor glycemic control, leads to a dysregulated metabolism, responsible for a long-term proinflammatory condition. In this scenario, an increasingly important role is played by chronic inflammation-induced oxidative stress that might concur with impaired glucose-associated conditions to promote tumour progression (Figure 1).

Hyperglycaemia is the hallmark for diabetes and results both from insufficient insulin production in pancreatic $\beta$ cells, as in T1D, and from the increase of systemic insulin resistance, as in T2D [23]. Some authors reported a direct effect of glycaemia on cancer initiation, proliferation, migration, and invasiveness [24], and extensive research is presently available supporting a causative link between IGT/T2D and BC [25-29], which translates into a mild risk of breast carcinogenesis (a 1.2 risk ratio in the meta-analysis by Larsson et al. [4]) especially among postmenopausal women [3032]. However, in patients with early-stage BC, T2D has been reported to be an independent predictor of lower BCspecific survival and, generally, of overall survival rates [33]. It is worthy to underline that the risk was independent of other conditions known to be associated with IR, such as obesity [32], thus supporting the involvement of site-specific mechanisms. These recognize, as a common denominator, the insulin axis. Insulin, in fact, is a potent regulator of human sexual steroid hormone synthesis that interferes with their signal transduction at cellular level $[34,35]$. Indeed, in T2D patients, high levels of insulin reduces in the concentration of circulating sex hormone binding protein (SHBG) [36], thus leading to an increase in the levels of bioactive oestrogens which are responsible for the proliferation of both breast and endometrial cells, for the inhibition of apoptosis [37] and, possibly, for the enhancement of hormonal carcinogenesis $[34,38]$. Furthermore, insulin and IGF-1 themselves are responsible for oestrogen production by enhanced expression of aromatase. Indeed, in obese T2D subjects, oestrone and estradiol are overproduced in the adipose tissue by the intense activity of aromatase [39], which has been found significantly expressed both in breast and in tumour tissues [40], and may fuel BC growth [38]. IGF-1, which participates in oestrogen receptor signalling via IGF-1 receptor/ER interaction, cooperates with oestrogens to regulate proliferation, apoptosis, and differentiation of mammary epithelial cells in a bidirectional way $[35,41]$. Furthermore, the interaction between IGF-1 and $17 \beta$-estradiol results in the proliferation of breast carcinoma cells [42]. Therefore, it appears evident that insulin and oestrogen might have a mutual interrelationship capable of conferring high risk for endocrine-related cancers, especially in postmenopausal women [41].

The insulin/IGF axis is deeply involved in diabetesassociated increased risk and progression of cancer, to such an extent that it has been demonstrated that cancer cells overexpress both insulin and IGF-1 receptors $[43,44]$. Physiologically, insulin exerts both metabolic and mitogenic 


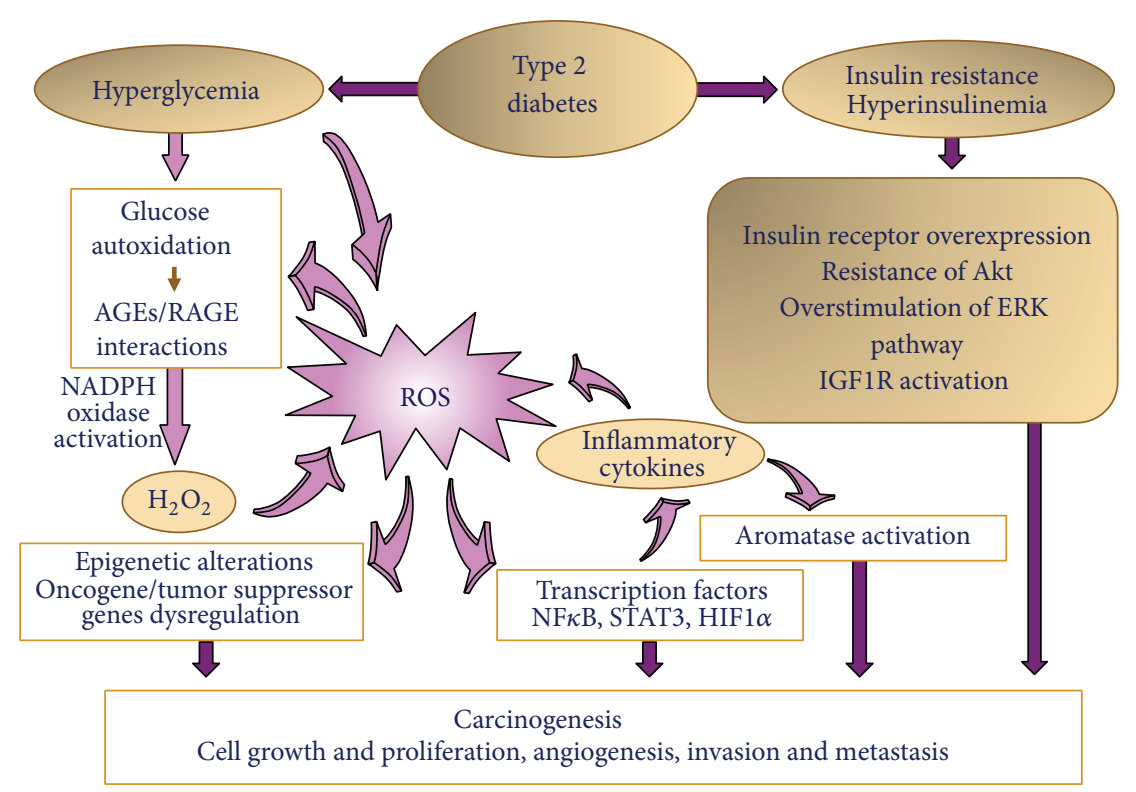

FIGURE 1: Pathways of oxidative stress associated with diabetes mellitus: mechanisms of carcinogenesis. Type 2 diabetes (T2D) causes both hyperglycaemia and hyperinsulinemia/IR. Hyperglycemia may induce reactive oxygen species (ROS) production directly via glucose metabolism and autooxidation and indirectly through the formation of advanced glycation end products (AGE) and their receptor (RAGE) binding. ROS, in turn, may exert their effects on DNA, through activation of signaling molecules (i.e., nuclear transcription factor- $\kappa \mathrm{B}-\mathrm{NF}-$ $\kappa \mathrm{B})$ and subsequent transcription of genes encoding cytokines and adhesive proteins. Hyperinsulinemia, insulin resistance (IR) and insulinlike growth factor-1 (IGF-1) activate signaling pathways, such as mitogen-activated protein kinase (MAPK) and AKT signaling pathways, that lead to carcinogenesis.

effects, the former being mediated by phosphatidylinositol 3-kinase (PI3-K) pathway [45], whereas the latter is mainly achieved through mitogen-activated protein kinase (MAPK) pathway [34]. IGF-1 shares similar mechanisms of action, particularly in hyperinsulinemic conditions. In BC, they rather act as mitogens to enhance tumourigenesis via either pathway: insulin, via the insulin receptor substrate 1 (IRS1) and IGF-1, by binding to its own receptor (IGF1R) [46] (Figure 1). It is worth noting that the activation of the $\mathrm{PI} 3 \mathrm{~K}$ pathways is required for insulin-induced upregulation of vascular endothelial growth factor (VEGF), which sustains neoangiogenesis and, thus, cancer progression [47]. Although the complex IGF-1/IGF1R shares high homology with insulin/insulin receptor [46], its activation results in a stronger favouring effect on BC cell proliferation and survival [48]. Due to this last evidence, in a condition of hyperinsulinemia, IGF1R can be activated both directly by the high circulating levels of insulin and, indirectly, through insulin-mediated upregulation of IGF-1 [48].

\section{Chronic Inflammation and Oxidant Stress in T2D Converge in Breast Cancer}

The consequences of hyperglycaemia on cancer cells behaviour can be either direct, as already reported, or indirect, through the increase in the levels of insulin/IGF-1 and inflammatory cytokines in circulation, such as interleukin-6 (IL-6) and tumour necrosis factor-alpha (TNF- $\alpha$ ) [49], together with the most classical inflammatory markers such as C-reactive protein [3], but also through oxidative stress generation [50] and platelet activation [51]. Indeed, the two pathways of inflammation and oxidative stress seem to converge in the activation of nuclear factor $\kappa \mathrm{B}$ $(\mathrm{NF} \kappa \mathrm{B})$ [52], signal transducer and activator of transcription 3 (STAT3), and hypoxia-inducible factor $1 \alpha$ (HIF1 $\alpha$ ) [53]. These conditions are accompanied by an increase in free radicals, which can damage lipids and DNA both directly and indirectly and concur to promote oxidative stress and to amplify the inflammatory process [53] (Figure 1).

A condition of oxidative stress or of altered redox system is established following an unbalance between the production of reactive oxygen species (ROS) and/or reactive nitrogen species (RNS) and their removal by endogenous antioxidants [54]. ROS, like superoxide radical $\left(\mathrm{O}_{2}{ }^{-}\right)$, the hydroxyl radical $\left(\mathrm{HO}^{-}\right)$, and the nonradical hydrogen peroxide $\left(\mathrm{H}_{2} \mathrm{O}_{2}\right)$, which normally participate to cell signalling, at high concentrations cause cell and tissue injury and damage. In T2D patients high levels of malondialdehyde (MDA), expression of the free radical-mediated lipid peroxidation, have been found associated with an increase in antioxidant enzymes possibly as a consequence of the adaptive response to prooxidant in diabetic state [55].

In this favorable milieu, ROS can initiate carcinogenesis by functioning as chemical effectors in the context of a redox unbalance [56], rendering cancer cells insensible to apoptosis, disrupting the cell anchorage sites and sustaining de novo angiogenesis [57]. It is widely acknowledged that ROS are key mediators of the metabolic coupling between the aerobic glycolysis in stromal cells (Warburg effect) and 
oxidative stress in cancer cells, which favours mitochondrial metabolism and tumourigenesis [58]. The changes in gene expression not resulting in DNA alteration, are commonly referred to as epigenetic and are regulated at several levels, among which are DNA methylation, histone modification and noncoding RNAs [53]. ROS have been implicated in both aberrant DNA hypermethylation and hypomethylation, and ROS-induced DNA methylation pattern alterations have been demonstrated both in malignant transformation and in cancer progression, thus representing important players in the epigenetic regulation in cancer cells (Figure 1) [59].

The association between T2D and cancer is further sustained by the elevated glycolytic rates and the formation of advanced glycation end products (AGEs) which, following interaction with their receptor (RAGE), leads to ROS generation, activation of NF- $\kappa \mathrm{B}$ and, finally, to cell damage (Figure 1). Conversely, the demonstration that blockade of RAGE-mediated signaling inhibits breast tumour growth and metastases, further evidences that RAGE expression is associated with BC [60].

As regards the inflammatory implications in $\mathrm{BC}$, it has been demonstrated that tumour cells highly express also IL- 8 , IL-1 $\beta$ and monocyte chemoattractant protein-1 (MCP$1)$, whose levels are associated with poor recurrence-free survival in patients with HER2(-) tumours [61] and, in general, with poorer prognosis $[50,62]$. Further evidence of a major role of cytokines in $\mathrm{BC}$ tumour progression comes from the demonstration that macrophages isolated from the tumour microenvironment of inflammatory BC patients secrete chemotactic cytokines that favour both dissemination and metastasis of carcinoma cells [63].

An important regulatory role is exerted by ROS in many cellular processes, among which the 3-phosphatase and tensin homolog (PTEN), which under normal conditions is a PI3-kinase inhibitor and, thus, acts on adhesion and motility [57]. It has been demonstrated that conditions of PTEN oxidation can result in BC promotion [57]. Genes encoding for proteins of the redox system are largely affected by oxidative stress, their polymorphism being responsible DNA damage, gene mutations, and, finally, carcinogenesis. The variations in genes from the stress oxidative pathway, such as polymorphism in exon 2 of the superoxide dismutase 2 (SOD2), catalase $(C A T)$, and endothelial NO synthase (eNOs) genes, are, at various levels, involved in BC development [64].

In $\mathrm{BC}$, a modulatory role has been established for the products of lipid peroxidation, hydroperoxides (HPs), 8isoprostanes, and MDA in cancer initiation and progression [92-94]. In addition and in further support of this statement, it has been reported that advanced stages of the disease are characterized by a more pronounced oxidative status than earlier stages, with a marked reduction of the antioxidant enzyme catalase activity and an enhanced lipid peroxidation together with higher nitric oxide levels [94]. Furthermore, in patients with BC, lipid peroxidation profiling at diagnosis was significantly correlated with a 5-year recurrence, following tumour removal, possibly leading to relapse or metastatic disease [94].

\section{Effects of Antidiabetic Treatment on Breast Cancer Initiation and Progression}

Of course, talking about the association between T2D and $\mathrm{BC}$ we must take into consideration the causative association between $\mathrm{T} 2 \mathrm{D}$ and $\mathrm{BC}$ deriving from clinical trials reporting the effects of antidiabetic medications. Insulin, insulin analogues and secretagogues (all acting through an increase of the circulating levels of insulin) have been associated with increased risk of cancer [95]. Insulin treatment, in particular, was investigated in a recent meta-analysis of 10 cohort studies, demonstrating a combined risk ratio (RR) of 1.28 (95\% CI: $1.03,1.59$ ) and individual RRs ranging from 1.19 to 3.87 [95]. However, when BC was investigated separately, inconsistent results were observed $[66,74]$. Other glucose-lowering drugs, such as sulfonylureas and glinides acting through sustained insulin production, have been investigated, but data are conflicting and deeper investigation is required to substantiate their possible association with increased cancer risks [95].

Metformin, in turn, was constantly associated with a reduced risk of cancer due not only to indirect mechanisms related to inhibition of hepatic gluconeogenesis and reduced insulin signaling via inhibition of phosphoinositide 3-kinase (PI3K) cellular response, but also to direct mechanisms operating through the tumour suppressor protein, LKB1, mediated activation of the AMP-activated protein kinase (AMPK) pathway and consequent suppression of energy stress response ultimately affecting cancer cell survival [96]. Indeed, in neoplastic cells, the increased AMPK activity leads to downstream inhibition of PI3K/Akt/mammalian target of rapamycin (mTOR) and MEK/ERK1/2mTOR signaling, protein synthesis, and proliferation [19]. Metformin can also directly inhibit tumour cell growth by modulating cyclin D1medicated cell cycle and the expression of tumour suppressor p53 in different tumour cells including breast carcinoma cells $[97,98]$. Another mechanism reported for cell apoptosis and death mediated by metformin, is by increasing oxidative stress, following AMPK and forkhead transcription factor 3 (FOXO3) protein activation [98], and by increasing activities of antioxidant molecules, such as $\mathrm{Cu}-\mathrm{Zn}$, SOD, catalase, and GSH in the erythrocytes [53]. As a consequence, this would render the erythrocytes less prone to oxidative stress [53].

The benefits of the reduction in insulin levels in T2D or hyperinsulinaemic cancer patients, whose tumour growth is under the influence of insulin, may account for the suggestion to use metformin in this subset of patients. The capability of metformin to increase apoptosis of BC cells in vitro has been documented in experimental models employing wild-type, tamoxifen-resistant, and oestrogen-deprived MCF-7 cells [99]. Interestingly, the reduction of neoplastic growth was more pronounced when metformin and tamoxifen were used in combination [99]. Thus, metformin-induced pleiotropic effects might be effective in enhancing the activities of the currently available hormonal therapies.

Overall, several meta-analysis of metformin clinical trials demonstrated a substantial reduction of cancer risk (approximately 40\%) compared with no use (Table 1) [73, 75-78]. However, when specific cancer sites were analyzed separately, reduced risk was confirmed in colorectal and 


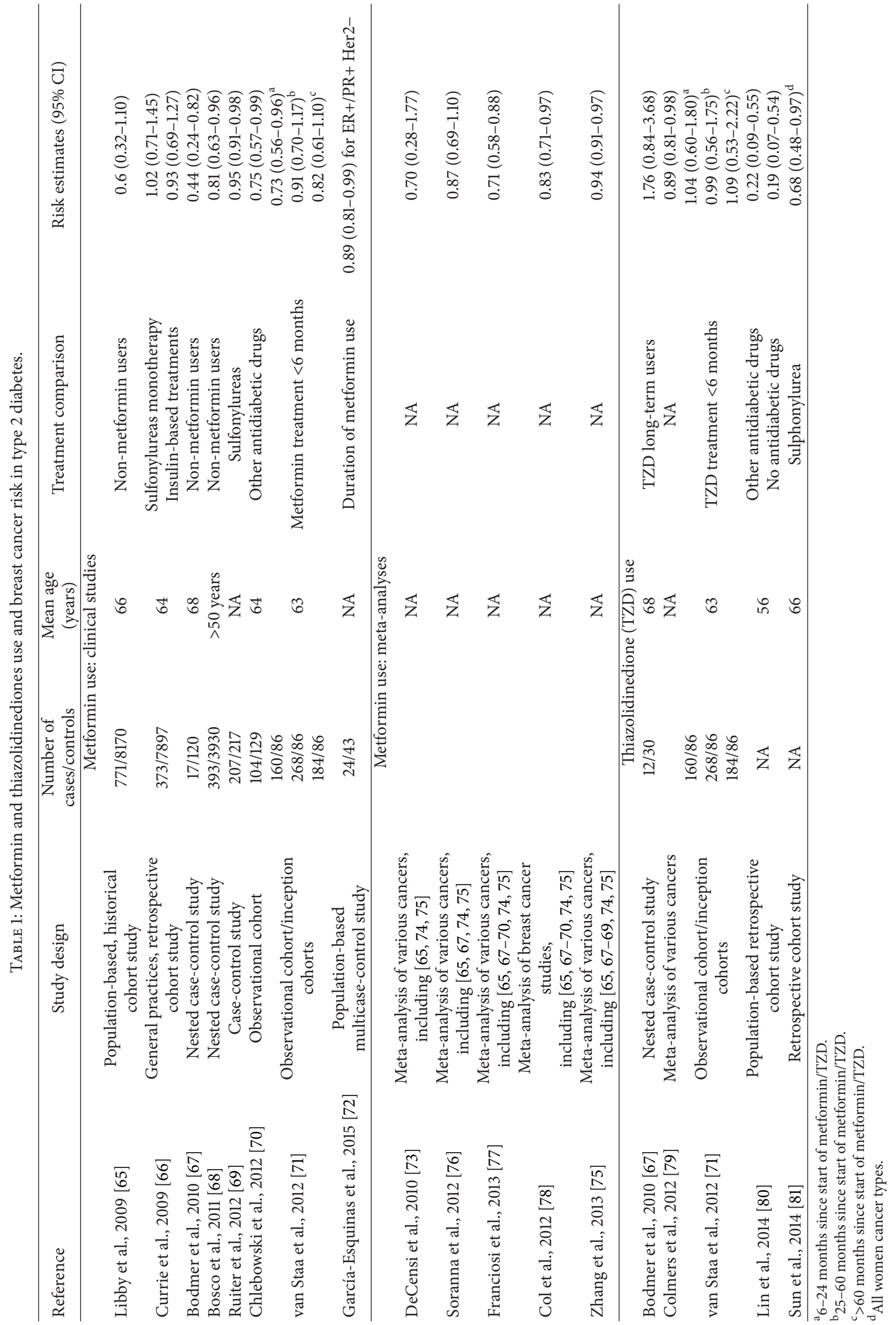




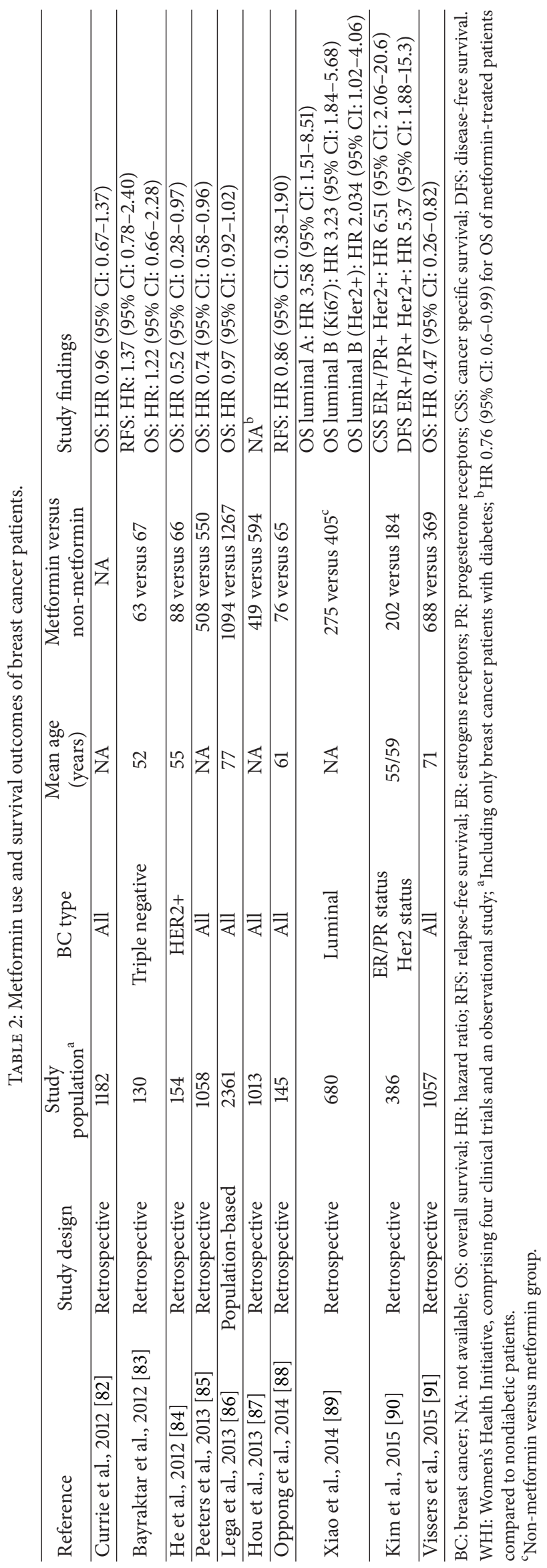


pancreatic cancers, whereas discordant results were reported for metformin use and $\mathrm{BC}$ risk [73, 75-78]. Looking into the single studies reported in the meta-analyses, pioneering retrospective investigations showed a nonsignificant association between metformin use and $\mathrm{BC}$ risk $[65,66]$, which was confirmed in subsequent case-control studies (Table 1) [6772 ], except when analyses were performed to estimate the impact of different duration metformin use [71]. Beside an increased BC risk, several evidences suggest a positive impact of metformin treatment on breast cancer survival outcomes, especially in particular subset of patients (Table 2) [82-91], at a point that this drug is currently being investigated for its effects on invasive disease-free survival and other outcomes in a phase III randomized trial in early BC (Clinical Trials.gov identifier: NCT01101438, estimated study completion date: December 2017).

Finally, thiazolidinediones (TZDs, namely, pioglitazone and rosiglitazone), introduced as oral antidiabetic agents to directly target insulin resistance, have been recently investigated for possible association with cancer risk, mainly because of the public health alert issued by the EMA concerning an increased risk of bladder cancer among diabetic patients treated with pioglitazone [100]. Despite this association, a meta-analysis performed by Colmers et al. demonstrated that TZDs use was related to a modest but significantly reduced risk of BC (RR: 0.89, 95\% CI 0.810.98 ) [79]. These results were confirmed in a recent six-year population-based cohort study showing a dose dependent decrease in specific cancer risks in diabetic patients using TZDs [80]. Furthermore, the results of a retrospective analysis of the electronic health record-based Cleveland Clinic Diabetes Registry cross-indexed with the histology-based tumour registry over an 8-year period, clearly demonstrated that TZDs use in women was associated with a $32 \%$ decreased cancer risk compared with sulphonylurea [81].

\section{Conclusions and Perspectives}

Looking back on what has been discussed, it appears clear that T2D should be looked at as a hidden enemy and that understanding the association between diabetes, oxidative stress, and site-specific cancers is becoming imperative and efforts should be employed to improve screening measures and to develop risk assessment tools. Oxidative stress, indeed, besides being responsible for the damage-induced development of cancer, represents a key steps involved in the mutagenesis that leads to carcinogenesis and might be responsible for the redox adaptation of cancer cells that become resistant to anticancer agents. This appears even more mandatory in an aging world in which sociodemographic, epidemiological, and technological factors are responsible for an increase in life expectancy and a higher health care demand for the National Health Systems (NHS). Older people, in fact, are more vulnerable to noncommunicable diseases (typically T2D and cancer) resulting in a considerable impact on the NHS, as more resources are needed to warrant appropriate standards of medical care and improve quality of life. In this context, the knowledge that the rate of biological aging is at least partially modulated by genes interacting with stressor exposures [101] is of utmost importance and, after more than 50 years, the mitochondrial free radical theory of aging is still endorsed [102]. Despite the conflicting outcomes of nontargeted antioxidants in clinical trials, there is growing evidence that helping with lifestyle interventions (including physical activity, dietary modifications, and appropriate therapeutic strategies, counting also supportive antioxidant supplementation ones) might have a clinically relevant role in reducing $\mathrm{BC}$ risk or progression in postmenopausal women with T2D. Finally, it becomes imperative for the clinicians to consider all comorbidities when dealing with diabetic patients with cancer, in which outcomes of both disease and chemotherapy may result in a poorer prognosis.

\section{Conflict of Interests}

The authors report no conflict of interests.

\section{Authors' Contribution}

Patrizia Ferroni and Silvia Riondino contributed equally to this paper.

\section{Acknowledgments}

This work has been partially supported by the Italian Ministry of Education Universities and Research Grant MERIT RBNE08NKH7. All authors report no potential conflict of interests with any company/organization whose products or services may be discussed in this paper. The authors wish to thank the nurses of the Medical Oncology Unit and to express deep gratitude to all patients and their families, who over the years have eagerly contributed to their research.

\section{References}

[1] International Diabetes Federation, IDF Diabetes Atlas, 6th edition, 2014, http://www.idf.org/diabetesatlas/.

[2] E. Giovannucci, D. M. Harlan, M. C. Archer et al., "Diabetes and cancer: a consensus report," Diabetes Care, vol. 33, no. 7, pp. 1674-1685, 2010.

[3] C. X. Xu, H. H. Zhu, and Y. M. Zhu, "Diabetes and cancer: associations, mechanisms, and implications for medical practice," World Journal of Diabetes, vol. 5, no. 3, pp. 372-380, 2014.

[4] S. C. Larsson, C. S. Mantzoros, and A. Wolk, "Diabetes mellitus and risk of breast cancer: a meta-analysis," International Journal of Cancer, vol. 121, no. 4, pp. 856-862, 2007.

[5] A. A. Onitilo, J. M. Engel, I. Glurich, R. V. Stankowski, G. M. Williams, and S. A. Doi, "Diabetes and cancer I: risk, survival, and implications for screening," Cancer Causes and Control, vol. 23, no. 6, pp. 967-981, 2012.

[6] Y. Handelsman, D. Leroith, Z. T. Bloomgarden et al., "Diabetes and cancer-an AACE/ACE consensus statement," Endocrine Practice, vol. 19, no. 4, pp. 675-693, 2013.

[7] E. Gouveri, N. Papanas, and E. Maltezos, "The female breast and diabetes," The Breast, vol. 20, no. 3, pp. 205-211, 2011.

[8] L. Vona-Davis and D. P. Rose, "Type 2 diabetes and obesity metabolic interactions: common factors for breast cancer risk 
and novel approaches to prevention and therapy," Current Diabetes Reviews, vol. 8, no. 2, pp. 116-130, 2012.

[9] M. G. Schrauder, P. A. Fasching, L. Häberle et al., "Diabetes and prognosis in a breast cancer cohort," Journal of Cancer Research and Clinical Oncology, vol. 137, no. 6, pp. 975-983, 2011.

[10] P. J. Goodwin, M. Ennis, K. I. Pritchard et al., "Insulin- and obesity-related variables in early-stage breast cancer: correlations and time course of prognostic associations," Journal of Clinical Oncology, vol. 30, no. 2, pp. 164-171, 2012.

[11] K. M. J. de Bruijn, L. R. Arends, B. E. Hansen, S. Leeflang, R. Ruiter, and C. H. J. van Eijck, "Systematic review and meta-analysis of the association between diabetes mellitus and incidence and mortality in breast and colorectal cancer," British Journal of Surgery, vol. 100, no. 11, pp. 1421-1429, 2013.

[12] M. Sant, C. Allemani, M. Santaquilani, A. Knijn, F. Marchesi, and R. Capocaccia, "Survival of cancer patients diagnosed in 1995-1999. Results and commentary," European Journal of Cancer, vol. 45, no. 6, pp. 931-991, 2009.

[13] P. Minicozzi, F. Berrino, F. Sebastiani et al., "High fasting blood glucose and obesity significantly and independently increase risk of breast cancer death in hormone receptorpositive disease," European Journal of Cancer, vol. 49, no. 18, pp. 3881-3888, 2013.

[14] L. A. Carey, C. M. Perou, C. A. Livasy et al., "Race, breast cancer subtypes, and survival in the Carolina Breast Cancer Study," The Journal of the American Medical Association, vol. 295, no. 21, pp. 2492-2502, 2006.

[15] A. A. Memon, L. Bennet, B. Zöller et al., "Circulating human epidermal growth factor receptor 2 (HER2) is associated with hyperglycaemia and insulin resistance2 (HER2)," Journal of Diabetes, vol. 7, no. 3, pp. 369-377, 2015.

[16] J. M. Fernndez-Real, J. A. Menendez, G. Frühbeck, J. M. Moreno-Navarrete, A. Vazquez-Martín, and W. Ricart, "Serum HER-2 concentration is associated with insulin resistance and decreases after weight loss," Nutrition and Metabolism, vol. 7, article 14, 2010.

[17] N. Vazzana, S. Riondino, V. Toto et al., "Obesity-driven inflammation and colorectal cancer," Current Medicinal Chemistry, vol. 19, no. 34, pp. 5837-5853, 2012.

[18] A. Marble, "Diabetes and cancer," The New England Journal of Medicine, vol. 211, no. 8, pp. 339-349, 1934.

[19] M. Pollak, "The insulin and insulin-like growth factor receptor family in neoplasia: an update," Nature Reviews Cancer, vol. 12, no. 3, pp. 159-169, 2012.

[20] E. Orgel and S. D. Mittelman, "The links between insulin resistance, diabetes, and cancer," Current Diabetes Reports, vol. 13, no. 2, pp. 213-222, 2013.

[21] H. Noto, A. Goto, T. Tsujimoto, K. Osame, and M. Noda, "Latest insights into the risk of cancer in diabetes," Journal of Diabetes Investigation, vol. 4, no. 3, pp. 225-232, 2013.

[22] R. C. Baxter, "IGF binding proteins in cancer: mechanistic and clinical insights," Nature Reviews Cancer, vol. 14, no. 5, pp. 329341, 2014.

[23] P. Vigneri, F. Frasca, L. Sciacca, G. Pandini, and R. Vigneri, "Diabetes and cancer," Endocrine-Related Cancer, vol. 16, no. 4, pp. 1103-1123, 2009.

[24] L. C. Richardson and L. A. Pollack, "Therapy insight: influence of type 2 diabetes on the development, treatment and outcomes of cancer," Nature Clinical Practice Oncology, vol. 2, no. 1, pp. 48-53, 2005.
[25] P. Muti, T. Quattrin, B. J. B. Grant et al., "Fasting glucose is a risk factor for breast cancer: a prospective study," Cancer Epidemiology Biomarkers and Prevention, vol. 11, no. 11, pp. 13611368, 2002.

[26] D. A. Lawlor, G. D. Smith, and S. Ebrahim, "Hyperinsulinaemia and increased risk of breast cancer: findings from the British women's heart and health study," Cancer Causes and Control, vol. 15, no. 3, pp. 267-275, 2004.

[27] M. J. Gunter, D. R. Hoover, H. Yu et al., "Insulin, insulin-like growth factor-I, and risk of breast cancer in postmenopausal women," Journal of the National Cancer Institute, vol. 101, no. 1, pp. 48-60, 2009.

[28] G. Chodick and I. Zucker, "Diabetes, gestational diabetes and the risk of cancer in women: epidemiologic evidence and possible biologic mechanisms," Women's Health, vol. 7, no. 2, pp. 227-237, 2011.

[29] V. Formica, M. Tesauro, C. Cardillo, and M. Roselli, "Insulinemia and the risk of breast cancer and its relapse," Diabetes, Obesity and Metabolism, vol. 14, no. 12, pp. 1073-1080, 2012.

[30] K. S. Peairs, B. B. Barone, C. F. Snyder et al., "Diabetes mellitus and breast cancer outcomes: a systematic review and metaanalysis," Journal of Clinical Oncology, vol. 29, no. 1, pp. 40-46, 2011.

[31] E. Weiderpass, G. Gridley, I. Persson, O. Nyrén, A. Ekbom, and H.-O. Adami, "Risk of endometrial and breast cancer in patients with diabetes mellitus," International Journal of Cancer, vol. 71, no. 3, pp. 360-363, 1997.

[32] G. C. Kabat, M. Kim, B. J. Caan et al., "Repeated measures of serum glucose and insulin in relation to postmenopausal breast cancer," International Journal of Cancer, vol. 125, no. 11, pp. 2704-2710, 2009.

[33] W.-W. Chen, Y.-Y. Shao, W.-Y. Shau et al., "The impact of diabetes mellitus on prognosis of early breast cancer in Asia," The Oncologist, vol. 17, no. 4, pp. 485-491, 2012.

[34] D. P. Rose and L. Vona-Davis, "The cellular and molecular mechanisms by which insulin influences breast cancer risk and progression," Endocrine-Related Cancer, vol. 19, no. 6, pp. R225R241, 2012.

[35] Z. Suba, "Interplay between insulin resistance and estrogen deficiency as co-activators in carcinogenesis," Pathology and Oncology Research, vol. 18, no. 2, pp. 123-133, 2012.

[36] B. Arcidiacono, S. Iiritano, A. Nocera et al., "Insulin resistance and cancer risk: an overview of the pathogenetic mechanisms," Experimental Diabetes Research, vol. 2012, Article ID 789174, 12 pages, 2012.

[37] M. Weichhaus, J. Broom, K. Wahle, and G. Bermano, "A novel role for insulin resistance in the connection between obesity and postmenopausal breast cancer," International Journal of Oncology, vol. 41, no. 2, pp. 745-752, 2012.

[38] E. J. Gallagher and D. LeRoith, "Diabetes, antihyperglycemic medications and cancer risk: smoke or fire?" Current Opinion in Endocrinology, Diabetes and Obesity, vol. 20, no. 5, pp. 485494, 2013.

[39] M. P. Cleary and M. E. Grossmann, "Minireview: obesity and breast cancer: the estrogen connection," Endocrinology, vol. 150, no. 6, pp. 2537-2542, 2009.

[40] W. R. Miller, "Aromatase and the breast: regulation and clinical aspects," Maturitas, vol. 54, no. 4, pp. 335-341, 2006.

[41] Y. Hawsawi, R. El-Gendy, C. Twelves, V. Speirs, and J. Beattie, "Insulin-like growth factor-oestradiol crosstalk and mammary gland tumourigenesis," Biochimica et Biophysica Acta, vol. 1836, no. 2, pp. 345-353, 2013. 
[42] C.-W. Lin, L.-Y. Yang, S.-C. Shen, and Y.-C. Chen, "IGF-I plus E2 induces proliferation via activation of ROS-dependent ERKs and JNKs in human breast carcinoma cells," Journal of Cellular Physiology, vol. 212, no. 3, pp. 666-674, 2007.

[43] A. V. Lee, P. Zhang, M. Ivanova et al., "Developmental and hormonal signals dramatically alter the localization and abundance of insulin receptor substrate proteins in the mammary gland," Endocrinology, vol. 144, no. 6, pp. 2683-2694, 2003.

[44] D. Sachdev and D. Yee, "The IGF system and breast cancer," Endocrine-Related Cancer, vol. 8, no. 3, pp. 197-209, 2001.

[45] J. Á. Fresno Vara, E. Casado, J. de Castro, P. Cejas, C. BeldaIniesta, and M. González-Barón, "P13K/Akt signalling pathway and cancer," Cancer Treatment Reviews, vol. 30, no. 2, pp. 193204, 2004.

[46] P. F. Christopoulos, P. Msaouel, and M. Koutsilieris, "The role of the insulin-like growth factor-1 system in breast cancer," Molecular Cancer, vol. 14, no. 1, article 43, 2015.

[47] Z. Y. Jiang, Z. He, B. L. King et al., "Characterization of multiple signaling pathways of insulin in the regulation of vascular endothelial growth factor expression in vascular cells and angiogenesis," The Journal of Biological Chemistry, vol. 278, no. 34, pp. 31964-31971, 2003.

[48] J. Riedemann and V. M. Macaulay, "IGF1R signalling and its inhibition," Endocrine-Related Cancer, vol. 13, supplement 1, pp. S33-S43, 2006.

[49] I. F. Godsland, "Insulin resistance and hyperinsulinaemia in the development and progression of cancer," Clinical Science, vol. 118 , no. 5, pp. 315-332, 2010.

[50] J. E. Goldberg and K. L. Schwertfeger, "Proinflammatory cytokines in breast cancer: mechanisms of action and potential targets for therapeutics," Current Drug Targets, vol. 11, no. 9, pp. 1133-1146, 2010.

[51] M. Murata, M. Mizutani, S. Oikawa, Y. Hiraku, and S. Kawanishi, "Oxidative DNA damage by hyperglycemia-related aldehydes and its marked enhancement by hydrogen peroxide," FEBS Letters, vol. 554, no. 1-2, pp. 138-142, 2003.

[52] P. Ferroni, S. Basili, A. Falco, and G. Davì, "Platelet activation in type 2 diabetes mellitus," Journal of Thrombosis and Haemostasis, vol. 2, no. 8, pp. 1282-1291, 2004.

[53] R. Wisastra and F. Dekker, "Inflammation, cancer and oxidative lipoxygenase activity are intimately linked," Cancers, vol. 6, no. 3, pp. 1500-1521, 2014.

[54] P. Ferroni, N. Vazzana, S. Riondino, C. Cuccurullo, F. Guadagni, and G. Davì, "Platelet function in health and disease: from molecular mechanisms, redox considerations to novel therapeutic opportunities," Antioxidants \& Redox Signaling, vol. 17, no. 10, pp. 1447-1485, 2012.

[55] A. Likidlilid, N. Patchanans, T. Peerapatdit, and C. Sriratanasathavorn, "Lipid peroxidation and antioxidant enzyme activities in erythrocytes of type 2 diabetic patients," Journal of the Medical Association of Thailand, vol. 93, no. 6, pp. 682-693, 2010.

[56] M. Matsuda and I. Shimomura, "Increased oxidative stress in obesity: implications for metabolic syndrome, diabetes, hypertension, dyslipidemia, atherosclerosis, and cancer," Obesity Research \& Clinical Practice, vol. 7, no. 5, pp. e330-e341, 2013.

[57] M. Parri and P. Chiarugi, "Redox molecular machines involved in tumor progression," Antioxidants \& Redox Signaling, vol. 19, no. 15, pp. 1828-1845, 2013.

[58] U. Martinez-Outschoorn, F. Sotgia, and M. P. Lisanti, "Tumor microenvironment and metabolic synergy in breast cancers: critical importance of mitochondrial fuels and function," Seminars in Oncology, vol. 41, no. 2, pp. 195-216, 2014.
[59] Q. Wu and X. Ni, "ROS-mediated DNA methylation pattern alterations in carcinogenesis," Current Drug Targets, vol. 16, no. 1, pp. 13-19, 2015.

[60] A. M. Korwar, H. S. Bhonsle, A. D. Chougale et al., "Analysis of AGE modified proteins and RAGE expression in HER2/neu negative invasive ductal carcinoma," Biochemical and Biophysical Research Communications, vol. 419, no. 3, pp. 490-494, 2012.

[61] Y. A. Cho, M.-K. Sung, J.-Y. Yeon, J. Ro, and J. Kim, "Prognostic role of interleukin-6, interleukin-8, and leptin levels according to breast cancer subtype," Cancer Research and Treatment, vol. 45, no. 3, pp. 210-219, 2013.

[62] C. A. Gilbert and J. M. Slingerland, "Cytokines, obesity, and cancer: new insights on mechanisms linking obesity to cancer risk and progression," Annual Review of Medicine, vol. 64, pp. 45-57, 2013.

[63] M. M. Mohamed, E. A. El-Ghonaimy, M. A. Nouh, R. J. Schneider, B. F. Sloane, and M. El-Shinawi, "Cytokines secreted by macrophages isolated from tumor microenvironment of inflammatory breast cancer patients possess chemotactic properties," International Journal of Biochemistry and Cell Biology, vol. 46, no. 1, pp. 138-147, 2014.

[64] P. Rodrigues, G. de Marco, J. Furriol et al., "Oxidative stress in susceptibility to breast cancer: study in Spanish population," BMC Cancer, vol. 14, no. 1, article 861, 2014.

[65] G. Libby, L. A. Donnelly, P. T. Donnan, D. R. Alessi, A. D. Morris, and J. M. M. Evans, "New users of metformin are at low risk of incident cancer: a cohort study among people with type 2 diabetes," Diabetes Care, vol. 32, no. 9, pp. 1620-1625, 2009.

[66] C. J. Currie, C. D. Poole, and E. A. M. Gale, "The influence of glucose-lowering therapies on cancer risk in type 2 diabetes," Diabetologia, vol. 52, no. 9, pp. 1766-1777, 2009.

[67] M. Bodmer, C. Meier, S. Krähenbühl, S. S. Jick, and C. R. Meier, "Long-term metformin use is associated with decreased risk of breast cancer," Diabetes Care, vol. 33, no. 6, pp. 1304-1308, 2010.

[68] J. L. F. Bosco, S. Antonsen, H. T. Sørensen, L. Pedersen, and T. L. Lash, "Metformin and incident breast cancer among diabetic women: a population-based case-control study in Denmark," Cancer Epidemiology Biomarkers and Prevention, vol. 20, no. 1, pp. 101-111, 2011.

[69] R. Ruiter, L. E. Visser, M. P. P. van Herk-Sukel et al., "Lower risk of cancer in patients on metformin in comparison with those on sulfonylurea derivatives: Results from a large population-based follow-up study," Diabetes Care, vol. 35, no. 1, pp. 119-124, 2012.

[70] R. T. Chlebowski, A. McTiernan, J. Wactawski-Wende et al., "Diabetes, metformin, and breast cancer in postmenopausal women," Journal of Clinical Oncology, vol. 30, no. 23, pp. 28442852, 2012.

[71] T. P. van Staa, D. Patel, A. M. Gallagher, and M. L. de Bruin, "Glucose-lowering agents and the patterns of risk for cancer: a study with the General Practice Research Database and secondary care data," Diabetologia, vol. 55, no. 3, pp. 654-665, 2012.

[72] E. García-Esquinas, E. Guinó, G. Castaño-Vinyals et al., "Association of diabetes and diabetes treatment with incidence of breast cancer," Acta Diabetologica, 2015.

[73] A. DeCensi, M. Puntoni, P. Goodwin et al., "Metformin and cancer risk in diabetic patients: a systematic review and metaanalysis," Cancer Prevention Research, vol. 3, no. 11, pp. 14511461, 2010.

[74] H. M. Colhoun and SDRN Epidemiology Group, "Use of insulin glargine and cancer incidence in Scotland: a study from the 
Scottish Diabetes Research Network Epidemiology Group," Diabetologia, vol. 52, no. 9, pp. 1755-1765, 2009.

[75] P. Zhang, H. Li, X. Tan, L. Chen, and S. Wang, "Association of metformin use with cancer incidence and mortality: a metaanalysis," Cancer Epidemiology, vol. 37, no. 3, pp. 207-218, 2013.

[76] D. Soranna, L. Scotti, A. Zambon et al., "Cancer risk associated with use of metformin and sulfonylurea in type 2 diabetes: a meta-analysis," The Oncologist, vol. 17, no. 6, pp. 813-822, 2012.

[77] M. Franciosi, G. Lucisano, E. Lapice, G. F. M. Strippoli, F. Pellegrini, and A. Nicolucci, "Metformin therapy and risk of cancer in patients with type 2 diabetes: systematic review," PLoS ONE, vol. 8, no. 8, Article ID e71583, 2013.

[78] N. F. Col, L. Ochs, V. Springmann, A. K. Aragaki, and R. T. Chlebowski, "Metformin and breast cancer risk: a metaanalysis and critical literature review," Breast Cancer Research and Treatment, vol. 135, no. 3, pp. 639-646, 2012.

[79] I. N. Colmers, S. L. Bowker, and J. A. Johnson, "Thiazolidinedione use and cancer incidence in type 2 diabetes: a systematic review and meta-analysis," Diabetes and Metabolism, vol. 38, no. 6, pp. 475-484, 2012.

[80] H. C. Lin, Y. T. Hsu, B. H. Kachingwe, C. Y. Hsu, Y. S. Uang, and L. H. Wang, "Dose effect of thiazolidinedione on cancer risk in type 2 diabetes mellitus patients: a six-year population-based cohort study," Journal of Clinical Pharmacy and Therapeutics, vol. 39, no. 4, pp. 354-360, 2014.

[81] G. E. C. Sun, B. J. Wells, K. Yip et al., "Gender-specific effects of oral hypoglycaemic agents on cancer risk in type 2 diabetes mellitus," Diabetes, Obesity and Metabolism, vol. 16, no. 3, pp. 276-283, 2014.

[82] C. J. Currie, C. D. Poole, and E. A. Gale, "The influence of glucose-lowering therapies on cancer risk in type 2 diabetes," Diabetes Care, vol. 35, no. 2, pp. 299-304, 2012.

[83] S. Bayraktar, L. F. Hernadez-Aya, X. Lei et al., "Effect of metformin on survival outcomes in diabetic patients with triple receptor-negative breast cancer," Cancer, vol. 118, no. 5, pp. 1202-1211, 2012.

[84] X. He, F. J. Esteva, J. Ensor, G. N. Hortobagyi, M.-H. Lee, and S.-C. J. Yeung, "Metformin and thiazolidinediones are associated with improved breast cancer-specific survival of diabetic women with HER2+ breast cancer," Annals of Oncology, vol. 23, no. 7, pp. 1771-1780, 2012.

[85] P. J. H. L. Peeters, M. T. Bazelier, P. Vestergaard et al., "Use of metformin and survival of diabetic women with breast cancer," Current Drug Safety, vol. 8, no. 5, pp. 357-363, 2013.

[86] I. C. Lega, P. C. Austin, A. Gruneir, P. J. Goodwin, P. A. Rochon, and L. L. Lipscombe, "Association between metformin therapy and mortality after breast cancer: a population-based study," Diabetes Care, vol. 36, no. 10, pp. 3018-3026, 2013.

[87] G. Hou, S. Zhang, X. Zhang, P. Wang, X. Hao, and J. Zhang, "Clinical pathological characteristics and prognostic analysis of 1,013 breast cancer patients with diabetes," Breast Cancer Research and Treatment, vol. 137, no. 3, pp. 807-816, 2013.

[88] B. A. Oppong, L. A. Pharmer, S. Oskar et al., "The effect of metformin on breast cancer outcomes in patients with type 2 diabetes," Cancer Medicine, vol. 3, no. 4, pp. 1025-1034, 2014.

[89] Y. Xiao, S. Zhang, G. Hou, X. Zhang, X. Hao, and J. Zhang, "Clinical pathological characteristics and prognostic analysis of diabetic women with luminal subtype breast cancer," Tumor Biology, vol. 35, no. 3, pp. 2035-2045, 2014.

[90] H. J. Kim, H. Kwon, J. W. Lee et al., "Metformin increases survival in hormone receptor-positive, Her2-positive breast cancer patients with diabetes," Breast Cancer Research, vol. 17, no. 1 , article $64,2015$.

[91] P. A. Vissers, C. R. Cardwell, L. V. van de Poll-Franse, I. S. Young, F. Pouwer, and L. J. Murray, "The association between glucoselowering drug use and mortality among breast cancer patients with type 2 diabetes," Breast Cancer Research and Treatment, vol. 150, no. 2, pp. 427-437, 2015.

[92] A. Gönenç, Y. Özkan, M. Torun, and B. Şmşek, "Plasma malondialdehyde (MDA) levels in breast and lung cancer patients," Journal of Clinical Pharmacy and Therapeutics, vol. 26, no. 2, pp. 141-144, 2001.

[93] C. Panis, V. J. Victorino, A. C. S. A. Herrera et al., "Differential oxidative status and immune characterization of the early and advanced stages of human breast cancer," Breast Cancer Research and Treatment, vol. 133, no. 3, pp. 881-888, 2012.

[94] A. C. Herrera, V. J. Victorino, F. C. Campos et al., "Impact of tumor removal on the systemic oxidative profile of patients with breast cancer discloses lipid peroxidation at diagnosis as a putative marker of disease recurrence," Clinical Breast Cancer, vol. 14, no. 6, pp. 451-459, 2014.

[95] M. Janghorbani, M. Dehghani, and M. Salehi-Marzijarani, "Systematic review and meta-analysis of insulin therapy and risk of cancer," Hormones and Cancer, vol. 3, no. 4, pp. 137-146, 2012.

[96] A. Leone, E. Di Gennaro, F. Bruzzese, A. Avallone, and A. Budillon, "New perspective for an old antidiabetic drug: metformin as anticancer agent.", Cancer treatment and research, vol. 159, pp. 355-376, 2014.

[97] I. B. Sahra, K. Laurent, A. Loubat et al., “The antidiabetic drug metformin exerts an antitumoral effect in vitro and in vivo through a decrease of cyclin D1 level," Oncogene, vol. 27, no. 25, pp. 3576-3586, 2008.

[98] E. A. I. F. Queiroz, S. Puukila, R. Eichler et al., "Metformin induces apoptosis and cell cycle arrest mediated by oxidative stress, AMPK and FOXO3a in MCF-7 breast cancer cells," PLoS ONE, vol. 9, no. 5, Article ID e98207, 2014.

[99] L. M. Berstein, W. Yue, J.-P. Wang, and R. J. Santen, "Isolated and combined action of tamoxifen and metformin in wildtype, tamoxifen-resistant, and estrogen-deprived MCF-7 cells," Breast Cancer Research \& Treatment, vol. 128, no. 1, pp. 109-117, 2011.

[100] B. Kermode-Scott, "Meta-analysis confirms raised risk of bladder cancer from pioglitazone," British Medical Journal, vol. 345, Article ID e4541, 2012.

[101] E. S. Epel and G. J. Lithgow, "Stress biology and aging mechanisms: toward understanding the deep connection between adaptation to stress and longevity," Journals of Gerontology Series A: Biological Sciences and Medical Sciences, vol. 69, no. 1, pp. S10-S16, 2014.

[102] D. F. Dai, Y. A. Chiao, D. J. Marcinek, H. H. Szeto, and P. S. Rabinovitch, "Mitochondrial oxidative stress in aging and healthspan," Longevity \& Healthspan, vol. 3, no. 1, article 6, 2014. 


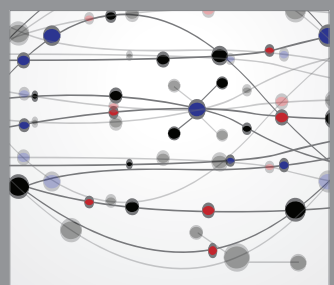

The Scientific World Journal
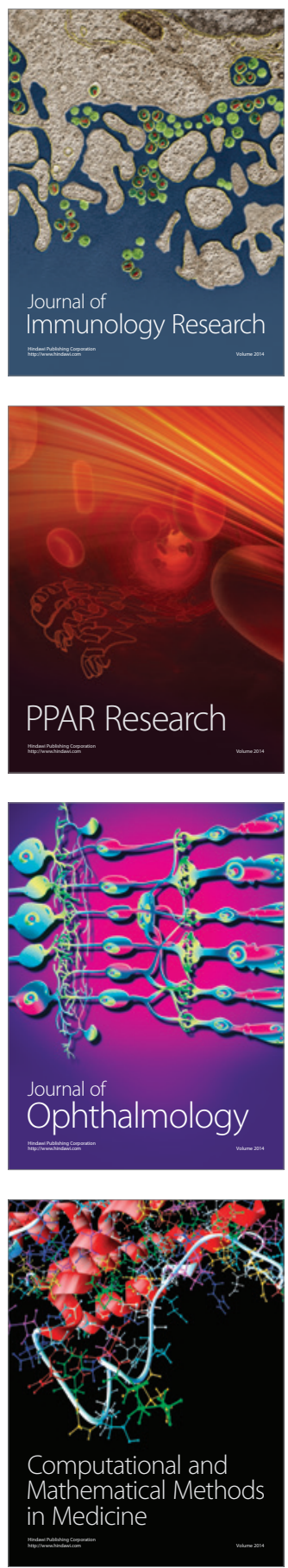

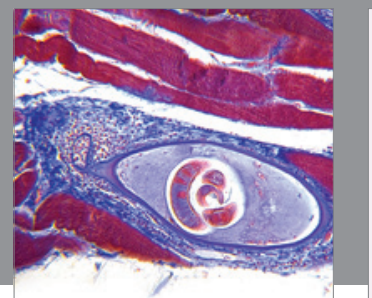

Gastroenterology

Research and Practice
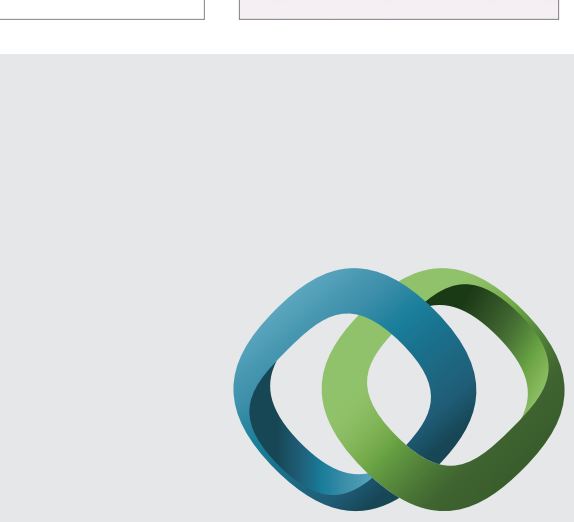

\section{Hindawi}

Submit your manuscripts at

http://www.hindawi.com
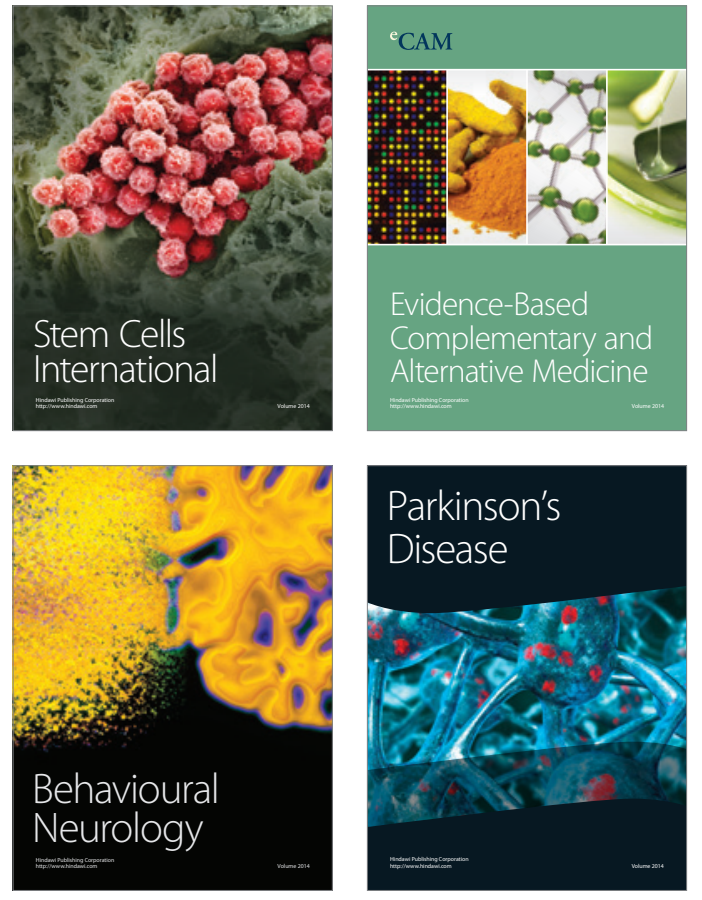
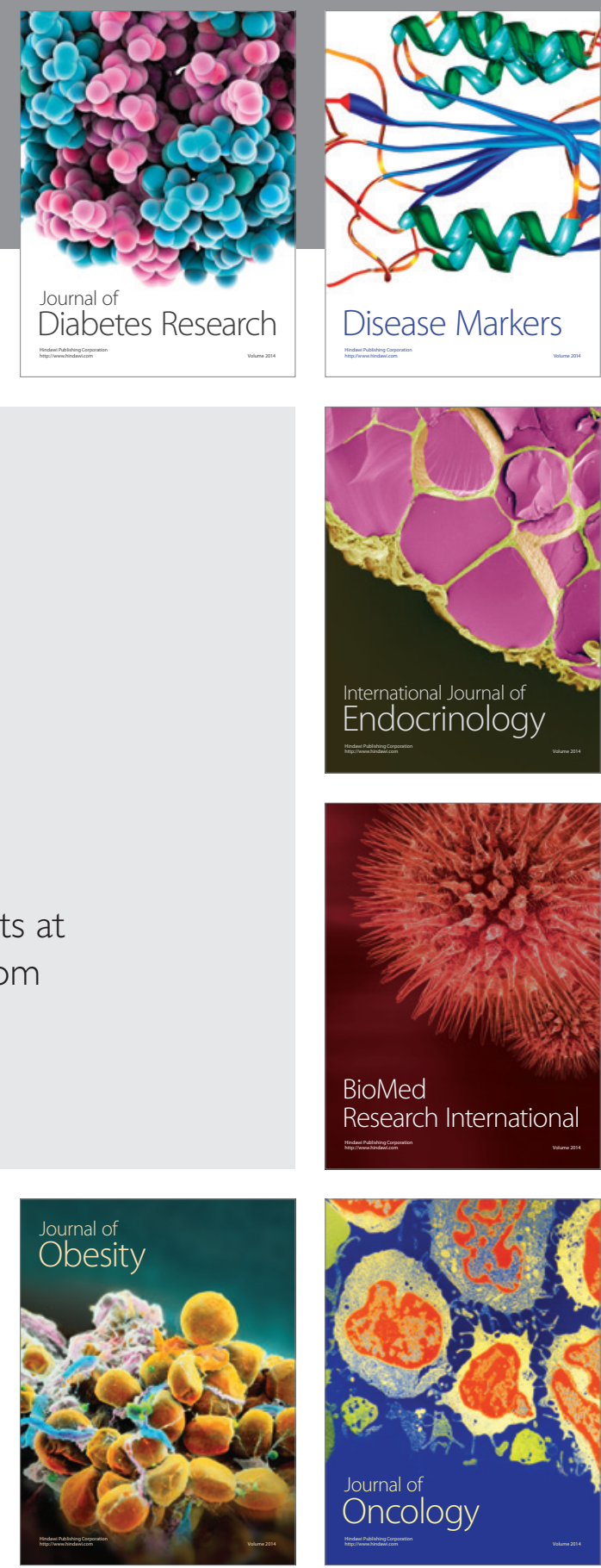

Disease Markers
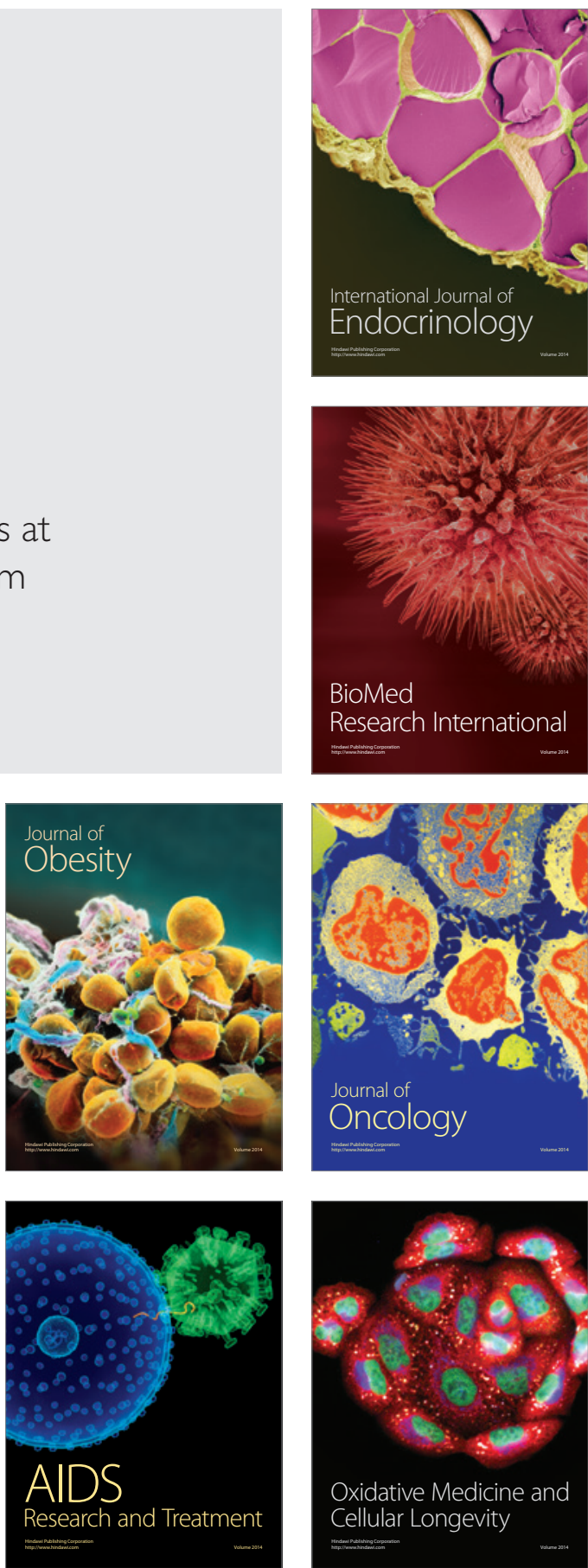